\title{
Soberanía alimentaria bajo nuevas lentes: Sinergias entre la nueva sociología económica y la resiliencia como un camino posible
}

Food sovereignty in a new lens: Synergies between new economic sociology and resilience as a possible way

Soberania alimentar sob novas lentes: As sinergias entre a nova sociologia econômica e a resiliência como um caminho possível

\section{Resumen}

Este artículo busca contribuir con en el debate sobre la soberanía alimentaria en los tiempos contemporáneos bajo la perspectiva de la Nueva Sociología Económica. Estas herramientas analíticas se utilizan para apoyar la interpretación de las nuevas formas de integración económica, así como las tradicionales, que, a su vez, están marginadas del proceso de modernización en un contexto de sociedad que constantemente produce crisis y riesgos. En este sentido, las formas de integración económica basadas en categorías como solidaridad, reciprocidad, cooperación y confianza pueden corroborar la construcción de resiliencia que contribuye para la constitución de la soberanía alimentaria.

Palabras chave: Soberanía alimentaria; Nueva sociología económica; Resiliencia; Sociedad de riesgo; Agroecología.

\begin{abstract}
This article seeks to contribute to the debate around food sovereignty in contemporary times from the perspective of the New Economic Sociology. These analytical tools are used here to support the interpretation of new forms of economic integration, as well as traditional ones, which are marginalized by the process of modernization in a context of society that constantly produces crises and risks. In this sense, forms of economic integration based on categories such as solidarity, reciprocity, cooperation and trust can corroborate the building of resilience that contributes to the constitution of food sovereignty.

Keywords: Food sovereignty; New economic sociology; Resilience; Risk society; Agroecology.

\section{Resumo}

O presente artigo busca aportar contribuições para o debate entorno da soberania alimentar na contemporaneidade sob a ótica da Nova Sociologia Econômica. Estas ferramentas analíticas são aqui utilizadas como apoio para a interpretação das novas formas de integração econômica, e também as tradicionais, que são marginalizadas pelo processo de modernização em um contexto de sociedade que produz crises e riscos constantemente. Nesse sentido, as formas de integração econômica baseadas em categorias como solidariedade, reciprocidade, cooperação e confiança, podem corroborar para a construção de uma resiliência que contribua para a constituição de uma soberania alimentar. Palavras-chave: Soberania alimentar; Nova sociologia econômica; Resiliência; Sociedade de risco; Agroecologia.
\end{abstract}

\section{Introducción}

En la sociedad moderna, el tema de la soberanía alimentaria ha venido adquiriendo nuevos y más profundos matices. Investigaciones recientes muestran la cuestión alimentaria va más allá de la dimensión de producción y comercialización de alimentos, llegando al concepto de soberanía alimentaria (Cassol \& Schneider, 2015). Este concepto toma su lugar en los debates por fuerza principalmente por la academia y los movimientos sociales como Vía Campesina Internacional. El tema también aparece en la Agenda 2030 de las Organizaciones Naciones Unidas (ONU) (Objetivos de Desarrollo Sostenible - 
2030), especialmente con relación al segundo objetivo que consiste en acabar con el hambre, promover la agricultura sostenible y lograr un sistema de soberanía alimentaria. En este contexto, la soberanía alimentaria propone una nueva concepción del consumo, que va más allá del tema rural y abarca a toda la población mundial.

El concepto de soberanía alimentaria se desarrolló en 1996, como resultado de la articulación de varios movimientos sociales campesinos (Hoyos \& D'Agostini, 2017). Estos movimientos sociales hicieron varias críticas a la Cumbre Mundial sobre la Alimentación, en particular sus políticas agrícolas neoliberales y el concepto de seguridad alimentaria adoptado por la Organización de las Naciones Unidas para la Alimentación y la Agricultura (FAO). Al comprender la seguridad alimentaria unicamente como un tema de la garantía de productos alimenticios, se marginan a varias otras variables clave, como el tema de dónde y cómo se producen los alimentos (Campos \& Campos, 2007). En contraste, los movimientos sociales, liderados por Vía Campesina, proponen el concepto de soberanía alimentaria:

Soberanía alimentaria es el derecho de cada nación para mantener y desarrollar su propia capacidad para producir los alimentos básicos de los pueblos, respetando la diversidad productiva y cultural. Tenemos el derecho a producir nuestros propios alimentos en nuestro propio territorio de manera autónoma. La soberanía alimentaria es una precondición para la seguridad alimentaria genuina. (Vía Campesina, 1996: sin página definida).

Esta conceptualización de la soberanía alimentaria se reafirmó en 2007, durante el Foro Mundial de Soberanía Alimentaria en Malí:

La soberanía alimentaria es el derecho de las personas a decidir su propio sistema de producción y alimentación, basado en alimentos saludables y culturalmente apropiados, producidos de manera sostenible y ecológica, lo que pone a quien los producen, distribuyen y consumen alimentos en el corazón de los sistemas y políticas alimentarias, por encima de las demandas de los mercados y las empresas, además de defender intereses e incluir a las generaciones futuras. (Foro Mundial para la Soberanía Alimentaria, 2007: sin página definida, traducción libre).

El acceso a alimentos saludables, de buena calidad y en cantidad suficiente es un requisito previo establecido por la soberanía alimentaria (Vía Campesina, 1996). Para esto, se traen al debate varios temas fundamentales, tales como: la transformación del sistema agrario y la energía, la genética, el agua y las cuestiones territoriales. Por lo tanto, el fortalecimiento de las prácticas sociales dentro del trabajo agrícola y la relación con la naturaleza se ha convertido en un tema esencial. Temas como la reforma agraria se unen y le dan otro significado al concepto de soberanía alimentaria, van más allá de la noción puramente social, y toman forma de una política centrada en la soberanía alimentaria de una región. En este sentido, la soberanía alimentaria busca garantizar las necesidades humanas, pero también romper con la lógica comercial y la explotación de la naturaleza, de modo a promover la reflexión sobre las condiciones de vida y de trabajo de los campesinos, además de la producción de alimentos de calidad, seguros, diversos, adecuados para la cultura local y ambientalmente sustentables (Hoyos \& D'Agostini, 2017).

Por lo tanto, varios autores, al problematizar el alcance de la seguridad alimentaria, cuestionan su lógica puramente comercial (Hoyos \& D'Agostini, 2017; Marques, 2010; Sage, 2014; Campos \& Campos, 2007). Sin embargo, estos estudios no explican con qué herramientas o con que apoyo teórico se puede poner frenos a la lógica estrictamente mercadológica. De ese modo, el presente artículo postula que la Nueva Sociología Económica (NSE) se constituyen una forma posible, capaz de articular este debate. La NSE es una corriente teórica que rompió con la comprensión económica clásica, producida en gran parte por Rational Choice Theory. La NSE desarrolló una comprensión de los actores inmersos en las redes sociales, de modo a exponer que no se puede estudiar las relaciones económicas fuera de este mundo social más amplio. Por otra parte, no considera a los individuos como meros receptores y transmisores de significados de las instituciones sociales. En otras palabras, no entiende a los actores como seres culturalmente incompetentes (Giddens, 2009), pero sí como sujetos sociales de 
los procesos de cambio social y económico.

Dicho esto, la NSE abre espacio para el análisis de categorías como solidaridad, reciprocidad, amistad, confianza y cooperación. Este enfoque puede apoyar una reflexión sobre la construcción de la resiliencia socioeconómica de grupos de agricultores familiares y/o agroecológicos, cuya trayectoria ha sido marcada por la evolución de los factores de vulnerabilidad. Esta resistencia, a su vez, es una categoría analítica que permite la reflexión de la capacidad de un sistema para absorber perturbaciones y reorganizarse a sí mismo mientras experimenta cambios, para retener esencialmente la misma función, estructura, identidad y retroalimentación (Walker, Holling, Carpenter \& Kinzig, 2004). Por lo tanto, la resistencia de esta comunidad la hace más fuerte frente a las crisis y los riesgos contemporáneos.

El presente trabajo se organiza en tres partes. La primera parte, con el título "Las interfaces de la soberanía alimentaria en la modernidad", busca ubicar las definiciones que rodean el concepto de soberanía alimentaria y su evolución a lo largo de los años desde la constitución de una sociedad moderna, en la que los riesgos (incluidos los alimentarios) se vuelven cada vez más latente. En la segunda parte, analizamos las contribuciones de la Nueva Sociología Económica, para estudiar las relaciones sociales de los individuos y qué puentes o sinergias se construyen entre la NSE y la resiliencia en grupos de agricultores locales, agricultores familiares o agroecológicos. Finalmente, el artículo construyó un diálogo entre la NSE y la resiliencia desarrollada por los agricultores, con el fin de pensar en las posibles formas que esta sinergia puede ofrecer para enfrentar los desafíos de la soberanía alimentaria, a partir de la hipótesis de que existe una construcción de resiliencia en nuevas formas de integración económica.

\section{Las Interfaces de la Soberanía Alimentaria en la Modernidad}

El debate sobre la soberanía alimentaria se ha constituido a lo largo del siglo XX, sufriendo los efectos del nuevo contexto social que surgió debido a las distintas revoluciones y crisis vinculadas a la modernidad. Los grandes cambios en la producción de alimentos, en gran parte como el resultado de las dos guerras mundiales y las innovaciones tecnológicas en la agricultura, reforzaron el surgimiento de políticas públicas de los estados nacionales, así como la creación de organismos internacionales como las Naciones Unidas y su brazo específicamente enfocado en el tema, la FAO (Chonchol, 2005).

En el período anterior a la Segunda Guerra Mundial, el debate sobre la soberanía alimentaria se redujo, en gran medida, a dos grupos distintos: 1) los científicos emergentes, que con avances científicos se centraron en la salud humana, advirtiendo sobre la falta de alimentos, desnutrición social y hambre; por lo tanto, defendían la necesidad de una mayor disponibilidad de alimentos. 2) los economistas de la escuela clásica, que vieron a los países pasar por un proceso de excedentes hasta la década de 1930, recomendando la reducción de la producción para resolver esta ecuación (Chonchol, 2005).

Con el advenimiento de la modernidad, el proceso de producción y comercialización de alimentos experimenta una transformación increíble, con una expansión continua, pero sin reflexionar sobre los riesgos que genera y por el agotamiento productivo y social que conlleva, produciendo efectos que incluso pueden hacer que su continuación sea inviable. Altiere (2010) describe este escenario como una "crisis leve". De esta manera, la Revolución Verde logró aumentar el volumen de producción de alimentos en todo el mundo, en gran parte debido al desarrollo tecnológico (Matos \& Pessôa, 2011). Sin embargo, las consecuencias y los efectos secundarios también se sienten. Además de la mayor concentración de tierras y el fortalecimiento de los monocultivos, la falta de tierras para el campesinado, el proceso de urbanización, el cambio climático, la desertificación y varios otros problemas sociales y ambientales también son perceptibles. Delgado llama la atención también para el "ajuste restringido de la orden globalizada", que es "básicamente caracterizado por restricciones externas e internas, expresadas por un alto endeudamiento público y dependencia externa" (Delgado, 2005: 62, traducción libre).

En este sentido, en el centro de la modernidad definido por Beck (1995) como la posibilidad de una autodestrucción 
creativa, en la cual el "sujeto" que causó esta destrucción no sería la revolución y mucho menos la crisis, pero el proceso de modernización en sí mismo puede reflexionar sobre los resultados de las características típicas de la Revolución. El dinamismo inherente a esta modernidad, considerada como reflexiva, es que dentro de las soluciones de un problema hay una semilla con un nuevo problema. Por lo tanto, la Revolución Verde, implementada en varios lugares del mundo, permitió un aumento en el volumen de alimentos producidos, pero esto resultó insuficiente para superar problemas estructurales en países considerados el Sur global. Además, la Revolución Verde, que viene en la ola de la modernidad, plantea varios problemas sociales y ambientales a escala mundial. Por ejemplo, la deforestación para el cultivo, la aparición de nuevas plagas y el uso excesivo de pesticidas, afectando los suelos, ríos y personas. Además de no resolver los problemas nutricionales y el hambre, como muchos argumentaron.

Las diversas transformaciones en el proceso de producción y de la comercialización agroalimentaria han demostrado su incapacidad de producir seguridad alimentaria y abastecimiento interno en los países en desarrollo, con la realidad del hambre como un problema actual. Un estudio publicado por la Organización de las Naciones Unidas para la Alimentación y la Agricultura (FAO), en 2016, mostró que aproximadamente una de cada nueve personas vive en la realidad del hambre. A la vez, las zonas rurales de los países en desarrollo han recurrido en gran medida al servicio de un sector agrícola de monocultivo de exportación. Productos como el maíz y la soya (como en el caso de Brasil) contribuyen significativamente para mantener el equilibrio económico de los países, lo que pesa sobre la balanza comercial nacional.

Un ejemplo de la importancia de la producción agrícola para los países en desarrollo es la extraordinaria expansión de la cosecha agrícola brasileña en el primer semestre de 2020 (según las estimaciones del Instituto Brasileño de Geografía y Estadística - IBGE), que se expandió un 8,7\% en relación con el año anterior En este sentido, la estimación es que 2020 alcanzará récords, con 246,7 millones de toneladas, incluso con la desaceleración económica causada por la pandemia de coronavirus (COVID-19). En enero, cuando la balanza comercial brasileña tuvo el mayor déficit en la historia, de US\$ 4.035 mil millones, el sector agrícola mantuvo un superávit de US\$ 5.12 mil millones.

Sin embargo, la Revolución Verde, por mucho que expanda la producción agrícola, tiene problemas tales como: degradación de la tierra y el suelo; proceso de desertificación; contaminación del agua; pérdida de biodiversidad; cambios climáticos; riesgo para la integridad del ecosistema; mayor vulnerabilidad a riesgos y plagas; dependencias de fertilizantes y pesticidas; estrés nutricional del suelo, impactos negativos en la salud pública y la calidad de los alimentos; concentración de la tierra; endeudamiento de los agricultores; y varios otros problemas económicos, ambientales y sociales (Altieri, 2010; Altieri \& Nicholls, 2012; Marques, 2010).

En este escenario, el tema de la soberanía alimentaria se fortalece y se convierte en el foco de debate de varios movimientos y organizaciones sociales, especialmente a partir de 1996, cuando ocurrió la Vía Campesina. Así, hubo un movimiento contrario a la lógica de la liberalización del sistema agroalimentario en el comercio internacional, apostando por una transición a las prácticas de producción agroecológica. En Brasil, el movimiento fue principalmente alrededor de la Vía Campesina, que fue la aglomeración del Movimiento de Trabajadores Rurales sin Tierra (MST), el Movimiento Afectado por la Presa (MAB) y el Movimiento de Pequeños Agricultores (MPA). Su objetivo era asociar la soberanía alimentaria con la solidaridad internacional (Vieira, 2008).

Para la FAO, "la seguridad alimentaria significa que las personas pueden producir o comprar alimentos suficientes para satisfacer sus necesidades diarias para llevar una vida activa y saludable". Por lo tanto, el concepto de soberanía alimentaria surge en contraste con el de seguridad alimentaria establecido por la FAO, ya que está directamente relacionado con un pueblo libre, que elabora sus propias políticas dirigidas a la producción y comercialización de alimentos, al mismo tiempo que garantiza que todas las personas tienen derecho a la alimentación, el respeto a su cultura alimentaria y la diversidad de formas de producción campesina. Desde esta perspectiva, el debate con respecto a la soberanía alimentaria busca calificar 
los mecanismos de seguridad alimentaria, lo que incluye temas como la reforma agraria redistributiva y la reestructuración del mercado. Todo con el objetivo de mejorar la distribución y el acceso a los alimentos (Wittman \& Blesh, 2017).

La necesidad de conformación sobre el concepto de soberanía alimentaria se debe a las diversas perspectivas sobre la noción de seguridad alimentaria, que pueden interpretarse de diferentes maneras. Desde una política dirigida a combatir el hambre a una acción que induzca una dieta equilibrada. Esta polisemia del término "seguridad alimentaria", a menudo, ignora las iniciativas centradas en la diversidad, la agricultura familiar y en los mercados locales. La soberanía alimentaria se opone a estos elementos y busca valorar la protección de la agricultura local. Por lo tanto, favorece temas como la agricultura familiar, la agroecología, los cortocircuitos alimentarios, la reforma agraria, el desarrollo territorial, entre otros (Altieri, 2010).

La agroecología, como método, puede mejorar la productividad y la sostenibilidad desde esta concepción de la soberanía alimentaria (Altieri, 2010). Esta sinergia entre ambos movimientos es entender que una región tiene el derecho de mantener y desarrollar su capacidad para producir diversos cultivos básicos y su cultura, al tiempo que enfatiza la necesidad de mercados locales, ciclos de consumo local y producción, acceso a tierra, semillas y agua; autonomía local; y algunos otros puntos.

Las diversas crisis experimentadas en los últimos años han demostrado la incapacidad de proporcionar alimentos a todos los pueblos, tanto en cantidad como en calidad. De esta forma, se desencadena la vulnerabilidad de los sistemas agroalimentarios (Marques, 2010). La subordinación de este concepto de soberanía alimentaria en la praxis diaria, y la adopción de una orientación modernizadora del área rural desde una lógica productivista, ha sido cuestionable en varios puntos, pero principalmente sus efectos sociales y ambientales. Para Altieri (1989), las crisis actuales demuestran los riesgos que plantea el sistema alimentario mundial, destacando las amenazas a la seguridad alimentaria de los pueblos. En este sentido, el autor destaca la degradación de los ecosistemas, la expansión de los monocultivos y expone sus externalidades socioambientales negativas.

El autor (1989) argumenta que se debe construir un paradigma alternativo de desarrollo de alimentos. Este nuevo paradigma debe apuntar a un sistema más equitativo y armonioso entre consumidores y productores. En este escenario, se propone la soberanía alimentaria como una alternativa saludable al actual sistema alimentario de riesgo. Por lo tanto, un gran número de teóricos ha estado defendiendo el diseño funcional de la soberanía alimentaria contra las desconexiones generadas por el actual sistema agroalimentario. Pensar en el estado de este sistema como resultado de una condición moderna es extremadamente importante, dada la confluencia de sus características de riesgo y la descrita por Beck (1995 y 2011) y Giddens (1995) como modernidad reflexiva.

De esta manera, la modernidad reflexiva puede entenderse aquí como una fase de la posibilidad de autodestrucción creativa de toda una era, en la cual un tipo de modernización siempre destruye y modifica otra (Beck, 1995). Esto configura la sociedad del riesgo dentro de la sociedad moderna, en la que los riesgos sociales, económicos, individuales y ambientales tienden cada vez más a ser universales y auto sostenibles. Así, en la sociedad de riesgos descrita por Beck (2011), el desarrollo tecnológico (que incluye los sistemas alimentarios y agrícolas con protagonismo) consolida el reconocimiento de la imprevisibilidad de las amenazas que genera. Por lo tanto, el concepto de sociedad de riesgo puede ser entendido como el de sociedad reflexiva, lo que significa que la sociedad moderna se convierte en un tema y un problema para sí mismo (Beck, 1995).

En esa línea, la Revolución Verde y el desarrollo tecnológico del sistema agroalimentario han demostrado ser insuficientes para reducir el hambre y la pobreza en el mundo, al tiempo que afectan directamente la biodiversidad ambiental y la diversidad de los cultivos. En la cuna de esta moderna sociedad reflexiva se encuentra la nueva matriz de producción agrícola, cuyo principal desafío es el desarrollo social equitativo, la conservación ecológica y la preocupación por la calidad de los alimentos de las personas. Ante esto, se presentarán, en el próximo tópico, las siguientes categorías de los análisis 
propuestos por la Nueva Sociología Económica, como una teoría que cuestiona la lógica económica mainstream y propone una nueva forma de ver el entorno de las interacciones sociales, destacando su capacidad de recuperación, que puede estar calificado en la defensa de la soberanía alimentaria como una ruta alternativa.

\section{Sinergias entre la NSE y la Resiliencia: un Posible Camino para el Debate sobre la Soberanía Alimentaria}

El sociólogo y antropólogo Marcel Mauss fue uno de los primeros autores de varios trabajos que sirvieron de base teórica para la NSE. El estudió los sistemas de intercambio en sociedades consideradas "primitivas", buscando entender como los intercambios económicos son relaciones que van más allá de la lógica económica. Al investigar este sistema de intercambio, el autor se da cuenta que al dar un objeto, la persona crea una obligación en relación con el individuo que lo dio. Este tipo de asociación produce fuertes alianzas y lazos de protección, que tienen un papel que va más allá de una simple búsqueda utilitaria de intereses individuales (Mauss, 2003).

En este sentido, Sabourin (2017) busca en la antropología económica de Mauss el concepto del don, que se opone al de intercambio mercantil. La reciprocidad marcada por los hechos de dar, recibir y devolver es el comienzo del ciclo del don. En esta perspectiva, la intención es expandir el concepto de reciprocidad, siendo como cualquier "acto con miras a crear un vínculo social" (Sabourin, 2017: 5. Traducción de los autores). A partir de esto, Sabourin argumenta que "la relación recíproca en una estructura bilateral simétrica genera un sentimiento de amistad; la relación de intercambio produce confianza y la división simétrica de los activos dentro de un grupo genera justicia” (Sabourin, 2017: 6. Traducción de los autores).

Sabourin construye su investigación empírica sobre el campesino y la reciprocidad en Brasil, abordando las relaciones de "ayuda" que desencadenan lazos sociales, sentimentales y simbólicos, en los que exigen una retribución igual, inmediata o diferida, y el beneficiario asumió una deuda. Por lo tanto, la reciprocidad y la redistribución como una acción distinta del intercambio, basada en una crítica de la definición de "economía" concebida por la elección racional. Este argumento del autor está tomado del trabajo de Polanyi, considerado uno de los emergentes de la Nueva Sociología Económica. Polanyi define la economía sustantiva "como un proceso instituido de interacción entre el hombre y su entorno que resulta en un suministro continuo de medios que satisfacen las necesidades materiales" (2012: 299. Traducción libre).

Esta relación recíproca (entre amigos, familiares, vecinos, entre otros) puede entenderse dentro de un marco de construcción de resiliencia de la población rural, frente a los desafíos y riesgos producidos por la sociedad moderna, considerando que Sabourin (2017) destaca que las leyes de intercambio tienen un predominio casi absoluto en las reglas económicas y políticas públicas. Hay un proceso de naturalización de estos intercambios como si fueran prácticas y normas universales, hecho que condujo el fortalecimiento del deseo de beneficio/ventajas, además del crecimiento de los intereses individuales de la sociedad. El autor también destaca que tanto la reciprocidad como los intercambios son modelos teóricos, y la realidad está formada por una mezcla. No tienes una sociedad con pura reciprocidad. Los dos modelos pueden coexistir de manera antagónica y con crisis, o pueden complementarse pacíficamente.

Granovetter, uno de los padres fundadores de Nueva Sociología Económica, también rescata en el trabajo de Polanyi (2012) el concepto de embeddedness (incrustación) que piensa en el mercado no como un agente externo, sino como una construcción política y social de la sociedad. Por lo tanto, las acciones del Estado, la historia, la cultura, las relaciones de clase y varios otros factores tienen el efecto de generar su propio orden institucional. A partir de esto, Granovetter argumenta que:

Los actores no se comportan como átomos fuera de un contexto social, ni adoptan servilmente un guión escrito para ellos por la intersección específica de categorías sociales que pueden ocupar. En cambio, sus intentos de llevar a cabo acciones con un propósito están inmersos en sistemas concretos y continuos de relaciones sociales. (2007: 8, traducción libre). 
Por lo tanto, Granovetter organiza todas las relaciones humanas, incluyendo la vida económica de los sistemas alimentarios, dentro de una relación social más amplia. El concepto de incrustación (o embeddedness) está respaldado por el entendimiento de que los actores tienen vínculos sociales, y cuanto más fuertes sean estos vínculos, mayor será la confianza mutua. En este caso, el autor afirma que la confianza también estaría relacionada con la presentación de recursos y con las relaciones desiguales de poder. Por ello, e la búsqueda de la soberanía alimentaria se convierte en un reto fundamental. Cómo son los lazos de confianza de los actores en la producción, y especialmente en la comercialización, y los lazos generados entre los individuos (productor y consumidor, cuando el intercambio se lleva a cabo en una relación directa, un ejemplo de los cuales son las ferias agroecológicas) que participan en las relaciones diarias amistades, confianza, reciprocidad, cooperación, solidaridad, poder y dependencia. Estos factores no pueden pasarse por alto en la constitución de la soberanía alimentaria.

A partir de esto, uno de los autores de la NSE que trabajó desde una perspectiva de mercado es Fligstein (2012). Este autor elaboró dos puntos de vista sobre el mercado como política. En el primero, el autor ce centra en cómo el Estado puede crear, a través de políticas públicas, canales de distribución para las áreas que se concentran. Pensar políticas públicas con un enfoque en la producción de espacios institucionales para el surgimiento y el apoyo de estos canales que fomentan las relaciones sociales, a menudo basadas en la reciprocidad y la confianza, que buscan categorías defendidas por la soberanía alimentaria. El Estado puede elegir esta ruta política alternativa, o no. Un ejemplo de esto es la elección de favorecer las grandes redes del sistema agroalimentario, en lugar de una política alimentaria alternativa difusa que priorice a los agricultores familiares.

La segunda visión del mercado como política es la gran crítica producida por Fligstein (2012) sobre la interpretación que la economía neoclásica hace sobre el actor (las personas). La economía neoclásica considera a los individuos como actores racionales, que siempre buscan maximizar sus intereses. En esta perspectiva, el autor entiende que las personas que viven en un "campo", tienden a mantener el sistema de dominación. Esta dominación es el resultado de una cultura local que influye en las relaciones sociales, que a su vez se compone de elementos cognitivos, que permiten a los actores interpretar sus espacios en las relaciones sociales constituidas.

La gran contribución de Fligstein (2007) a la Nueva Sociología Económica es pensar en los actores con capacidad de movilización y en construcción, que logran crear nuevos patrones de relación e interacción, basados en la cooperación. En este sentido, Fligstein argumenta que los individuos son mucho más que reproductores de las estructuras sociales en las que se encuentran. Esta relación dinámica nos permite pensar en las posibilidades de alcanzar la soberanía alimentaria, al pensar en la movilidad de los actores, que logran transformar las relaciones de poder en las que se establecen los roles sociales.

En esta perspectiva, los individuos son dotados de agencia, ya que tienen un panorama más amplio de iniciativas y no están subordinados automáticamente a una estructura social. Dotado de una habilidad social, las personas podrían desarrollar la cooperación, sin dejar de lado que se encuentran en un sistema de distribución desigual de recursos. Por lo tanto, la Nueva Sociología Económica, al estudiar los intercambios y las relaciones económicas, ubica a los individuos y sus relaciones sociales en el centro de análisis. El abordaje postula e que las relaciones económicas están inmersas/incrustadas en una compleja red social, distanciándose de la comprensión adoptada por la Teoría de la Elección Racional.

La NSE reafirma que las relaciones de reciprocidad, cooperación, amistad e interdependencia guían constantemente a las personas. No se puede ignorar que la sociedad moderna, como se describió anteriormente, está insertando un estilo cada vez más impersonal y complejo de relación económica. Por lo tanto, las relaciones económicas, como las ferias agroecológicas o el mercado local, tienden a constituirse como una acción de resistencia frente a la estandarización impuesta por la modernización del estilo de producción y comercialización, fortalecida en gran medida por la Revolución Verde.

El concepto de resiliencia, a su vez, aparece en el siglo XIX dentro del campo científico de la física, con el objetivo de describir el proceso en el que ciertos materiales tienen que volver al equilibrio después de sufrir una mutación (Folke, 2006). 
Recientemente, en la década de 1970, el concepto de resiliencia es absorbido por la ecología, más específicamente en sus sistemas socioecológicos, en oposición al paradigma de estabilidad ecológica (Holling, 1973). Sin embargo, otras áreas del conocimiento se apropiaron del concepto de resiliencia a fines del siglo XX, son: antropología, ciencias sociales, ciencias políticas, geografía y otras (Adger, 2000; Zzimmerer, 1994).

En este sentido, el concepto de resiliencia se describe ahora como la capacidad de renovarse, reorganizarse y desarrollarse ante las perturbaciones (Folke, 2006). Otros autores abordan la posibilidad de aprender y adaptar a los actores después de los eventos de crisis (Carpenter, Walker, Anderies \& Abel, 2001), o incluso su capacidad para producir nuevas oportunidades de desarrollo e innovación (Folke, 2006). Sin embargo, Adger (2006) también argumenta que los sistemas vulnerables pueden verse fuertemente afectados por disturbios menores, conflictos y crisis. Desde la perspectiva de Adger (2006), es necesario pensar en formas de fortalecer la resiliencia de una comunidad (como los agricultores familiares y/o agroecológicos, por ejemplo) ante los eventos adversos que pueden afectarla y, por lo tanto, enfrentar los eventos críticos como crisis económicas, ambientales, sociales y políticas, entre otros, para mantener esencialmente las mismas funciones, estructura, identidad y retroalimentación.

La agricultura familiar y/o agroecológica tiene una cierta capacidad de recuperación en su interior, dado que logra mantener y recuperar sus características fundamentales a la vez que son objetivos de restricciones que buscan transformar sus estructuras esenciales de relación (Milando, 2007; Jerneck y Olsson, 2008; Groenlandia y Szabolcs, 1994). Las transformaciones en las sociedades modernas tienen un impacto en estos grupos que están en la base de la producción y comercialización (agroecológica o no), sin embargo, su capacidad de recuperación les permite lograr una auto-reproducción de la forma de ser y producir, evitando transformaciones sociales más repentinas. En consecuencia, la continuidad de ciertas costumbres, prácticas, formas de producción, entre otras, tiende a ser más fuerte y superponer transformaciones y rupturas. Este fenómeno se puede notar durante la Huelga de Camioneros en Brasil (comenzó el 21 de mayo de 2018 hasta el 1 de junio de 2018), cuando la circulación de productos y bienes se vio obstaculizada en todo Brasil, sin embargo, los pequeños agricultores (agroecológicos o no) que venden alimentos en los sistemas de circuitos cortos se han fortalecido y ganado importancia en el suministro de alimentos.

En la siguiente sección, se busca demostrar cómo estas percepciones, producidas por la NSE, se pueden combinar con el marco conceptual aportado por la categoría analítica 'resiliencia' para una mejor comprensión del interior de los grupos de productores y comerciantes que integran el sistema agroalimentario. Por lo tanto, este movimiento busca contribuciones a los problemas y desafíos que enfrenta la soberanía alimentaria en los tiempos modernos.

\section{Contribuciones de la NSE y de la Resiliencia para Abordar los Desafíos de la Soberanía}

\section{Alimentaria}

Altieri (2010), al estudiar los agroecosistemas, señaló que las relaciones basadas en valores tradicionales tienden a ser menos vulnerables a pérdidas catastróficas. Eso se explica por la poca o ninguna dependencia de los insumos de alta tecnología, de las herramientas modernas y la variada producción de cultivos hacen que estos grupos sociales estén más preparados y sufran, proporcionalmente, menos con los impactos de las crisis. La defensa de pequeñas unidades productivas con biodiversidad, contra grandes propiedades de monocultivos, se debe a su capacidad y a la mitigación de los efectos de las catástrofes. En esta perspectiva, las unidades rurales diversificadas, las unidades agrícolas familiares y especialmente aquellas que son unidades agroecológicas, tienen una estructura que produce y sufre mucho menos problemas sociales (Marques, 2010). Es importante señalar que algunas crisis que resultan en la limitación de movilidad de la población (como el COVID19 y la huelga de los camioneros en Brasil en 2018) presentan ventajas los circuitos cortos de comercialización, que aparte de ser menos dependientes de petróleo, son marcados por lazos fuertes entre productor y consumidor, generando una relación de co- 
responsabilidad.

Sin embargo, la sociedad de riesgo creada por la modernización de los patrones de vida y consumo hace que los riesgos sean cada vez más inminentes, constantes y que afecten a todos (Beck, 2011). La agricultura considerada convencional, a menudo, en lugar de crear resiliencia y contribuir a la distribución equitativa de los alimentos, termina produciendo vulnerabilidades, especialmente para los países pobres o en desarrollo, cuya matriz económica se convierte, en la mayoría de los casos, en la venta de productos de bajo valor agregado al exterior, en un proceso en el cual los agricultores y productores son cada vez más vulnerables a las fluctuaciones de precios en el mercado. Además, otra vulnerabilidad que se está construyendo es la incapacidad de innovar, en la cual los países periféricos importan tecnologías maduras y exportan productos con menor valor agregado. La vulnerabilidad se constituye así en la no adquisición de conocimiento e innovación, que mantiene a estos países siempre en la periferia del centro. Por lo tanto, la importancia de pensar en los procesos de construcción de resiliencia está justificada.

Las unidades agrícolas pequeñas y diversas tienden a resistir a las transformaciones de la vida moderna, mostrándose capaces de innovar (siempre cuando apoyadas por servicios de extensión rural) y conectarse a mercados de cortocircuito, en los que existe la posibilidad de encontrar un mayor valor agregado a sus productos y menos vulnerabilidad (o mayor capacidad de recuperación) frente a la dinámica incierta del mercado. Esta perspectiva acompaña a la debatida por la soberanía alimentaria, que refuerza efectivamente estas referencias de calidad alimentaria ancladas en formas de producción más sostenibles y en el origen más local de los productos, lo que permite revalorizar la actividad agrícola.

Por lo tanto, la Nueva Sociología Económica permite comprender, de manera más amplia, las relaciones económicas involucradas entre estos agricultores familiares y/o agroecológicos, que establecen lazos sociales y económicos que van más allá de la lógica de la sociedad moderna. Al poner en el centro del debate categorías como solidaridad, reciprocidad, cooperación y amistad, la NSE propone una antítesis o nuevas formas para las relaciones económicas entendidas desde el homo economicus (hombre económico), que siguió a una comprensión del actor estrictamente racional.

Las pautas de la sociedad moderna (como el individualismo, la vida tecnológica y la impersonalidad en las relaciones) están ganando cada vez más hegemonía en los tiempos contemporáneos. Sin embargo, varios estudios muestran, desde el NSE, una perspectiva, en cierto sentido, contra hegemónica en grupos sociales agroecológicos o solo en agricultores familiares (Sabourin, 2009; Sabourin, 2017; Santos, 2004). Pensar en las relaciones contra hegemónicas como una acción de resiliencia de estos grupos de agricultores, en vista de las diversas posibilidades que impone la comprensión de las políticas públicas con la génesis de la Revolución Verde.

La resiliencia de mantener ciertas prácticas tradicionales, o incluso buscar nuevas prácticas contrahegemónicas, a menudo no se puede entender desde una lógica racional moderna. Es necesario considerar las inmersiones en las que se insertan los individuos, pensando en sus lugares en un universo de signos y representaciones. Según lo propuesto por la NSE, pensar en los vínculos sociales y las redes dentro de los grupos sociales es esencial para comprender las acciones de los individuos. 
Figura 1: Mapa conceptual.

\section{SOBERANIAA ALIMENTARIA}

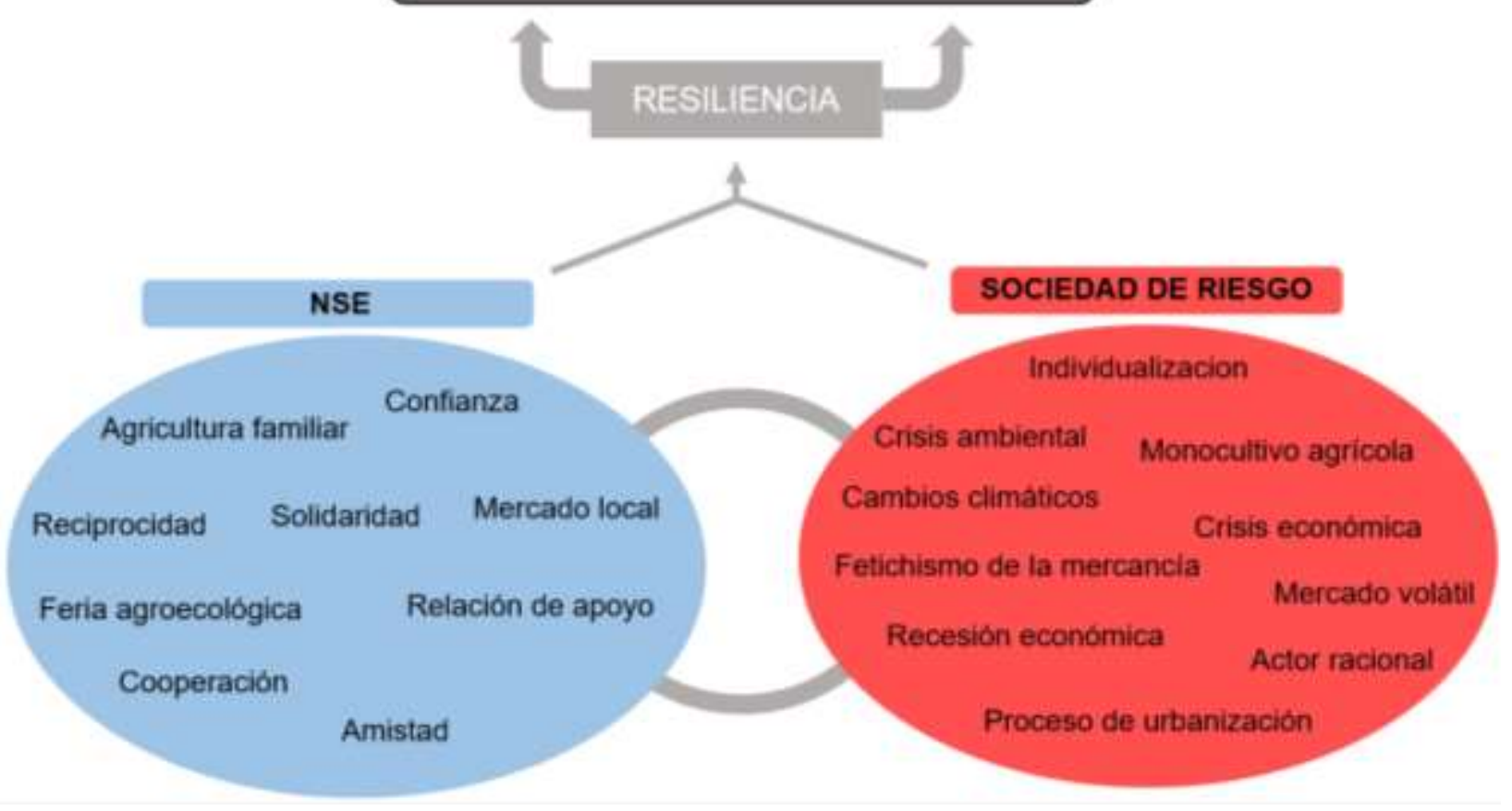

Fuente: Elaboración propia (2019).

La Imagen 1 describe cómo la capacidad de recuperación de las personas e instituciones median en categorías como la confianza, la reciprocidad, la cooperación, la amistad, el mercado local, entre otros, con los riesgos generados en la sociedad moderna. Y cómo todo este proceso ayuda en la formación y consolidación de la soberanía alimentaria de una sociedad. El esfuerzo producido aquí fue relacionar esa capacidad de recuperación de los pequeños agricultores, como una posibilidad de contribuir a las pautas de soberanía alimentaria, en vista de sus luchas y defensas. Asimilar las posibilidades analíticas de la NSE, para estudiar las posibilidades de resiliencia de los grupos agrícolas locales, al mismo tiempo que son fundamentales para la contribución de la soberanía alimentaria, desde varios puntos, que pueden destacarse: la transición agroecológica, una mayor estabilidad del mercado y autonomía de los agricultores.

\section{Consideraciones Finales}

Este artículo buscó entender cómo la articulación de las contribuciones conceptuales de la Nueva Sociología Económica y la resiliencia puede ser una forma posible de pensar sobre la forma en que la discusión de las diferentes formas de integración económica puede integrarse en la discusión sobre la soberanía alimentaria. Buscó explorar las ventajas y beneficios de estas sinergias entre las diversas formas de integración económica y la construcción de resiliencia entre los actores involucrados en la producción y el consumo en los sistemas agrícolas de alimentos.

El escenario de modernización resultó en un contexto de producción de riesgo con respecto a la producción y consumo de alimentos. Por esta razón, se destaca, como resultado fundamental, que la comprensión de las diversas formas de integración económica, incluidas las tradicionales, que fueron marginadas por el proceso de modernización puede corroborar la discusión sobre la construcción necesaria de resiliencia en la cadena de consumo y para la garantía de la soberanía alimentaria.

No se trata de jerarquizar una contribución teórica según la otra, sino de ilustrar cómo los fenómenos observados, 
defendidos y descritos (en las formas de producción, comercialización e interacción social, que parten de una noción contrahegemónica) por la nueva sociología económica pueden mostrar que el tejido social logra generar resiliencia frente a las formas convencionales de producción, en este caso, formas de producción como la agroecología y la agricultura familiar, nos permite ver formas que ayudan en la soberanía alimentaria, y aún más contra una generación de riesgo.

Por lo tanto, el artículo construyó un diálogo entre la NSE y la resiliencia desarrollada por los agricultores, para luego pensar en las posibles formas que esta sinergia puede ofrecer para enfrentar los desafíos de la soberanía alimentaria, en base a la hipótesis de que hay una construcción de resiliencia en las formas de integración económica cubierta por la NSE. El artículo comenzó una discusión conceptual, articulando estas categorías y teorías, pero los estudios empíricos que articulan estas mismas categorías pueden traer muchas posibilidades para avanzar en la frontera del conocimiento.

\section{Referencias}

Adger, W. (2000). Social and ecological resilience: are they related? Progress in Human Geography, 24 (3), $347-364$.

Adger, W. (2006). Vulnerability. Global Environmental Change, 16 (3), 268-281.

Ahn, C. \& Muller, A. (2019). South Korea: Ground Zero for Food Sovereignty and Community Resilience. Foreign Policy In Focus. https://www.thenation.com/article/south-korea-ground-zero-food-sovereignty-and-community-resilience/.

Altieri, M. (2010). Agroecologia, agricultura camponesa e soberania alimentar. Revista NERA, 13 (16), 22-32.

Altieri, M. (1989). Agroecologia: as bases científicas da agricultura alternativa. PTA/FASE.

Altieri, M. \& Nicholls, C. (2012). Agroecología: Única esperanza para la soberanía alimentaria y la resiliencia socioecológica. Agroecología, 7 (2), 65-83.

Andrade, J. G. de, Silva, M. G., Filho, F. de S. O. \& Feitosa, S. dos S. (2020). Diagnosis of creole seed production and storage techniques in rural settlements in Aparecida, Paraíba, Brazil. Research, Society and Development, 9 (5), 1-19.

Azevedo, L. L. T. \& Silva, I. L. (2019). Desenvolvimento Territorial sob a Perspectiva da Nova Sociologia Econômica. O Eco da Graduação, 4 (1), 5-23.

Beck, U. (2011). Sociedade de risco: rumo a uma outra modernidade. Editora 34.

Beck, U., Giddens, A. \& Lash, S. (1995). Modernização reflexiva. Unesp.

Carpenter S., Walker B., Anderies M. \& Abel N. (2001). From Metaphor to Measurement: Resilience of What to What? Ecosystems, 4 (1), $765-781$.

Campos, C. S. S. \& Campos, R. S. (2007). Soberania Alimentar como alternativa ao agronegócio no Brasil. Revista Electrónica de Geografía e Ciencias Sociales, Universidad Barcelona, 11 (245).

Cassol, A. \& Schneider, S. (2015). Produção e consumo de alimentos: novas redes e atores. Lua Nova, 1 (95), 143-180.

Chonchol, J. (2005). A soberania alimentar. Estudos Avançados, 19 (55), 33-49.

Dalbianco, A. B., Seabra Júnior, S., Santi, A., Trento, D. A., Dipple, F. L., Vendruscolo, M. C. \& Vendruscolo, M. V. (2020). The food crisis and the price of agricultural products. Research, Society and Development, 9 (11), 120-168.

Delgado, G. (2005). A questão agrária no Brasil, 1950-2003. Em: Jaccoud, L. (Ed.), Questão Social e Políticas Sociais no Brasil Contemporâneo (pp 51-90). Brasília: IPEA.

Duarte, B. M. (2019). Implementação de políticas públicas: as contribuições da nova sociologia econômica para uma perspectiva relacional. Sociedade Em Debate, 25(2), 139-154.

Fligstein, N. (2007). Habilidade social e a teoria dos campos. ERA, 47 (2), 61-81.

Fligstein, N., Dauter, L. (2012). A sociologia dos mercados. Caderno CRH, Salvador, 25 (66), 481-504.

Folke, C. (2006). Resilience: The emergence of a perspective for social-ecological systems analyses. Global Environmental Change, 16 (3), $253-267$.

Foro Mundial pela Soberania Alimentar. (2007). Malí. Declaração de Nyélény. Recuperado de: http:www.nyeleni.org/spip.php?article327.

Giddens, A. (2009). A Constituição da sociedade. Martins Fontes.

Granovetter, M. (1985). Ação Econômica e Estrutura Social. O problema da incrustação. Em: Marques, R. \& Peixoto, J. (Eds.), A nova sociologia econômica. Oeiras: Celta.

Greenland, D. \& Szabolcs, I. (Eds.). (1994). Soil resilience and sustainable land use. Wallingford: Cab International.

Holling, C. (1973). Resilience and Stability of Ecological Systems. Annual Review of Ecology and Systematics, 4, 1-23. 
Hoyos, C. \& D'Agostini, A. (2017). Segurança Alimentar e Soberania Alimentar: convergências e divergências. Revista NERA, 35, 174-198.

Jerneck, A. \& Olsson, L. (2008). Adaptation and the poor: development, resilience and transition. Climate Policy, 8 (2), 170-182.

Knickel, K., Redman, M., Darnhofer, I., Ashkenazy, A., Chebach, T., Šūmane, S., Tisenkopfs, T., Zemeckis, R., Atkociuniene, V., Rivera, M., Strauss, A., Kristensen, L., Schiller, S., Koopmans, M. \& Rogge, E. (2018). Between aspirations and reality: Making farming, food systems and rural areas more resilient, sustainable and equitable. Journal of Rural Studies, 59, 197-210.

Mann, A. (2018). Contesting Power: Food Sovereignty as Pedagogical Practice and Resistance. Food and Powe, Dublin Gastronomy Symposium, 1-8.

Marques, P. E. (2010). Embates em torno da segurança e soberania alimentar: estudo de perspectivas concorrentes. Segurança Alimentar e Nutricional, 17 (2), 78-87.

Matos, P. \& Pessôa, V. L. S. (2011). A modernização da agricultura no brasil e os novos usos do território. Geo UERJ, 22 (2), $290-322$.

Mauss, M. (2003). Ensaio Sobre a Dádiva. Em: Mauss, M. Sociologia e Antropologia. Cosac \& Naify.

Milando, J. (2007). Desenvolvimento e Resiliência Social em África: dinâmicas rurais de Cabinda. Centro de Estudos Africanos do ISCTE.

Polanyi, K. (2012). A subsistência do homem. Em: Polanyi, K. A subsistência do homem e ensaios correlatos. Contraponto.

Ramos, C. de M., Noda, H. \& Martins, A. L. U. (2021). Indigenous food security and sovereignty in the extreme north of Amapá - Brazil. Research, Society and Development, 10 (5), 1-19.

Sabourin, E. P. (2009). Comunidades camponesas e organização social da produção. Em: Sabourin, E. P. Camponeses do Brasil: entre a troca mercantil e a reciprocidade. Rio de Janeiro: Editora Garamond.

Sabourin, E. P. (2017). Produção camponesa e seguridade alimentar: uma análise pela teoria da reciprocidade. Revista Latinoamericana de Estudios Rurales, 2 (3), 1-21.

Sage, C. (2014). The Transition Movement and Food Sovereignty: From Local Resilience to Global Engagement in Food System Transformation. Journal of Consumer Culture, 14 (2), 254-275.

Santos, A. B. (2004). Necessidade de uma economia solidária: a visão de Karl Polanyi sobre os mercados. ResearchPapers in Economics (RePEc).

Vía Campesina. (1996). Soberanía alimentaria un futuro sin hambre. Em: Fórum De Ong Para La Soberanía Alimentaria, Roma. Declaración dirigida a la Cumbre Mundial de la Alimentación, Itália.

Vieira, F. B. (2008). Via Campesina: um projeto contra-hegemônico? Anais do III Simpósio Lutas Sociais na América Latina, 1-12.

Walker, B., Holling, C., Carpenter, S. \& Kinzig, A. (2004). Resilience, Adaptability and Transformability. Ecology and Society, 9 (2), 1-5.

Walsh-Dilley, M., Wolford, W. \& MCCarthy, J. (2016). Rights for resilience: food sovereignty, power, and resilience in development practice. Ecology and Society, 21 (1), 1-11.

Wittman, H. \& Blesh, J. (2017). Food Sovereignty and Fome Zero: Connecting Public Food Procurement Programmes to Sustainable Rural Development in Brazil. Journal of Agrarian Change, 17 (1), 81-105.

Zimmerer, K. S. (1994). Human Geography and the "New Ecology": The Prospect and Promise of Integration. Annals of the Association of American Geographers, 84 (1), 108-125. 